\title{
Fast Texture Segmentation Based on Semi-Local Region Descriptor and Active Contour
}

\author{
Nawal Houhou ${ }^{1, *}$, Jean-Philippe Thiran ${ }^{1}$ and Xavier Bresson ${ }^{2}$ \\ ${ }^{1}$ Signal Processing Laboratory 5, Ecole Polytechnique Fédérale de Lausanne (EPFL), \\ Lausanne, Switzerland. \\ 2 Department of Mathematics, University of California, Los Angeles, \\ CA 90095-1555, USA.
}

Received 26 March 2009; Accepted (in revised version) 22 July 2009

\begin{abstract}
In this paper, we present an efficient approach for unsupervised segmentation of natural and textural images based on the extraction of image features and a fast active contour segmentation model. We address the problem of textures where neither the gray-level information nor the boundary information is adequate for object extraction. This is often the case of natural images composed of both homogeneous and textured regions. Because these images cannot be in general directly processed by the gray-level information, we propose a new texture descriptor which intrinsically defines the geometry of textures using semi-local image information and tools from differential geometry. Then, we use the popular Kullback-Leibler distance to design an active contour model which distinguishes the background and textures of interest. The existence of a minimizing solution to the proposed segmentation model is proven. Finally, a texture segmentation algorithm based on the Split-Bregman method is introduced to extract meaningful objects in a fast way. Promising synthetic and real-world results for gray-scale and color images are presented.
\end{abstract}

AMS subject classifications: 65M10, 78A48

Key words: Semi-local image information, Beltrami framework, metric tensor, active contour, Kullback-Leibler distance, split-Bregman method.

\section{Introduction}

Texture segmentation is among the most challenging problems in image segmentation. The problem already begins with the definition of textures. The human eye can easily recognize different textures, but it is quite difficult to define them in mathematical terms. Then, there is a deliberate vagueness in the definition of textures, which explains the difficulty to conceptualize a model able to describe it. Besides, textures raise the problem of non-existence of significant edges and the non-homogeneity of intensity distributions

*Corresponding author. Email addresses: nawal.houhou@epfl.ch (N. Houhou), jean-philippe. thiran@epfl.ch (J.-P. Thiran), xbresson@math.ucla.edu (X. Bresson) 
lying in images. However, it is consensually admitted (e.g. [3]) that textures are fine scale-details, usually with some periodicity and oscillatory nature.

Different approaches deal with the extraction of homogeneous features from textures. Among these texture descriptors, some are statistical-based or transformed-based as the structure tensor $[8,54]$ or the Gabor filters [38]. Some recent methods propose to use the Beltrami framework [47], [27]. In this paper we propose a new method for the extraction of homogeneous regions or textures based on the Beltrami framework and on semi-local image information. We will see that our texture feature is more robust with respect to noise than the one we have previously presented in [27].

Once the feature extraction step is completed, a fast and robust algorithm to perform texture segmentation/extraction is needed. To reach this objective, we develop an algorithm based on the active contour model. In this image segmentation method, an initial contour is subjected to a speed term which drives the contour toward the boundary of the object to be segmented. The speed term is determined by the minimization of an energy functional composed of a contour smoothing term (internal energy) and an attraction term that pulls the contour towards the object boundaries (external energy). Active contour models have a long history. This method was originally introduced by Kass et al. in [31]. Then, Caselles et al. in [10] and Kichenassamy et al. in [32] proposed an intrinsic geometrical model, called geodesic/geometric active contours, where the curve evolution is handled by the level set method introduced by Osher and Sethian in [40]. Thus, the first generation of active contour segmentation methods [10,31,32] was based on edge detection. However, boundary-based segmentation is often too limited in many applications such as in medical image segmentation where fuzzy contours can be encountered or in natural images with textures. To deal with these problems, segmentation models based on region descriptors such as mean $[14]$ or variance $[29,55]$ have been developed. Among the region descriptors, the probability density function (pdf) (or histogram) is proven to be an efficient and general region descriptor.

Zhu and Yuille in [56] and Paragios, Rousson and Deriche in [41,45] approximated the pdf of the image regions by mixture of Gaussians, each one representing a homogeneous intensity region to be segmented. Jehan-Besson, Aubert, Barlaud, Faugeras and Herbulot in $[1,26,29]$ used the pdf of evolving regions of interest as a general region descriptor. The regions of interest are given by the minimization of region-based energies using the shape derivative tool defined by Delfour and Zolesio in [16]. Aubert, Barlaud, Faugeras and Jehan-Besson proved in [1] that minimizing a region-based functional with the shape derivative tool is equivalent to minimizing boundary-based functional with the calculus of variations. These authors applied in $[1,29]$ the shape derivation tool to the image segmentation problem. Along the same lines, Herbulot et al. in [26] used the shape derivative tool and information theoretical concepts (entropy and mutual information) to also perform image segmentation.

All these methods enable us to find local minimizers of the segmentation problem. Local minimizers mean that the quality of the segmentation results depends on the choice of the initial condition. In other words, the initial contour is critical to get satisfactory segmentation results, and to avoid bad local minimizers. The authors of $[5,12]$ proposed to refor- 
mulate the active contour problem in a convex optimization framework, providing a global minimizer to the active contour model. Besides, a fast minimization scheme ${ }^{\dagger}$ based on a dual approach of the TV norm and a splitting technique was introduced in [5], defining a fast segmentation algorithm. In [27], an unsupervised segmentation method based on the Kullback-Leibler distance and on non-parametric estimation of the pdf was introduced and solved with the fast minimization scheme defined in [5]. Recently, a new technique based on the Split-Bregman (SB) method [24] has been introduced to solve the convexified active contour model in a faster way than [5]. More precisely, this new minimization algorithm is as least as fast as graph-cuts, is more accurate because it uses isotropic schemes and does not have memory requirements in $3 \mathrm{D}$. We can notice that the SB method is actually equivalent to the augmented Lagrangian method $[49,51]$. In this paper, we propose to apply the SB method defined in [24] to solve the unsupervised segmentation problem. Finally, the main contributions of this paper are summarized as follows:

1. Definition of an intrinsic texture descriptor based on the Beltrami representation, semi-local image information and the metric tensor.

2. Proof of the existence of a minimizing solution for our variational segmentation problem in the space of functions with bounded variation.

3. Definition of a fast and accurate numerical scheme to solve the texture segmentation problem.

The outline of this paper is as follows. Section 2 studies the feature extraction problem for textures. Section 2.1 describes state-of-the-art feature extraction techniques. Then, Section 2.2 presents the new texture descriptor based on semi-local information in the Beltrami representation. Section 3 presents the active contour unsupervised segmentation method. Our segmentation method is then introduced in Section 3.1, followed by a mathematical justification in Section 3.2. Section 4 presents the convexification of the active contour energy and also introduces a fast numerical scheme based on the Split-Bregman method. In Section 5, experimental results on both texture gray-scale and color images are shown.

\section{Texture features}

This section aims at defining a pertinent feature descriptor for textures. The quality of texture segmentation methods is highly dependent of the quality of texture features. It is thus essential to define a texture feature as good as possible for the segmentation task.

\subsection{Image features}

The first and most natural texture feature is the image itself. For a scalar image $I$ : $\mathbb{R}^{2} \rightarrow \mathbb{R}$, each pixel is characterized by its gray-level value or intensity. If the image is composed of multiple channels (such as color images), then each pixel is described by

${ }^{\dagger}$ We mean the optimization scheme is faster than the Level Set method. 
a vector of intensities. When the image is composed of textures, then the pixel intensity value does not really give pertinent information. In fact, textures cannot be analyzed at the local pixel scale but need to be analyzed at a higher level scale, scale where information of textures is pertinent. Besides the intensity information, texture descriptors should consider the scales and the orientations (or lack of orientation) of the image. A natural approach for texture segmentation is thus to first represent the textured image by feature descriptors and then to apply a vector-valued segmentation scheme. It is clear that the quality of the segmentation will depend on the extraction of good discriminative features. We hereafter describe some of these features.

\subsubsection{Filter-based features}

A very popular class of texture features are the filter-based features of the given image. Filters such as the gradient filter or the wavelet bank filter [37] have been used for texture feature extraction and image segmentation (for examples $[44,47]$ ). Texture features generated by Gabor/Morlet wavelet transform [35] are powerful tools to discriminate textures of different orientations and scales. The main motivation is based on the fact that simple cells in the visual cortex can be modeled by Gabor functions [38]. The Gabor functions are parameterized by a wavelet orientation angle $\theta$ and a scale $\sigma$. Given a certain number of orientations and scales, the original image can be reconstructed from Gabor filter responses obtained by convolution of the given image and the set of $(\theta, \sigma)$-parameterized Gabor functions. Obviously, increasing the number of orientations and scales will improve the reconstruction quality. However, a good reconstruction can also be achieved by selecting only the most relevant filter responses s.a. in [28].

\subsubsection{Semi-local information-based feature}

As we said, the choice of features is difficult and critical to get an optimal segmentation result. A recent promising image feature to represent and process textures is the image intensity patch around the current pixel. The information on a close neighborhood around the current pixel is extracted and leads to semi-local information at each pixel. The patch idea as a feature vector was first introduced for texture synthesis $[17,18,36]$. Later, Buades et al. in [9] proposed to denoise images based on patch differences and non-local averaging. Gilboa and Osher in [22] proposed a non-local denoising model based on a variational framework. Finally, Bresson and Chan in [4] defined a variational unsupervised segmentation method also based on patch differences.

\subsubsection{Feature defined in the Beltrami framework}

Another way to represent textures is to use the Beltrami representation introduced by Sochen, Kimmel and Malladi in [50]. Sochen et al. proposed a new geometric representation of images by considering images as Riemannian manifolds embedded in a higher dimensional space. For instance, a standard 2 dimensional gray-value image $I: \mathbb{R}^{2} \rightarrow \mathbb{R}$ can be viewed as a surface $\Sigma$ with local coordinates $(x, y)$ embedded in $\mathbb{R}^{3}$ by a mapping 
$X:(x, y) \rightarrow\left(X_{1}=x, X_{2}=y, X_{3}=I(x, y)\right)$. This manifold-based representation of images offers two main advantages. Firstly, it allows using efficient tools borrowed from differential geometry to perform different image processing tasks such as denoising $[33,50]$ or segmentation $[6,47]$. The second main advantage of this framework is to work with arbitrary $N$ dimensional images. For example, a color image can be represented in a 5 dimensional space with the mapping $X:(x, y) \rightarrow\left(X_{1}=x, X_{2}=y, X_{3}=R(x, y), X_{4}=\right.$ $\left.G(x, y), X_{5}=B(x, y)\right)$, where R, G,B stands for red, green and blue. In [47], Sagiv, Sochen and Zeevi used the Beltrami framework to represent the texture image as a 2-D dimensional manifold embedded in a space of $N+2$ dimensions, where $N$ is the number of Gabor responses. Then, the first fundamental form (e.g. [34]), also called metric tensor, of the $(N+2)$-D texture manifold allowed them to define an intrinsic edge detector (see also [48]). The idea of using the metric tensor to intrinsically define the edges between different texture regions is natural and well-posed in the context of differential geometry. Indeed, the first fundamental form describes the distortion or rate of change of the manifold and so can detect boundary between different parts of the manifold corresponding to different homogeneous textures. Based on the metric tensor, Sagiv et al. used the geodesic active contour model [10] to drive the contour toward the boundary between two different textured regions by considering the edge detector function or stopping function as the inverse of the determinant of the metric tensor. To illustrate this idea, let consider the case of a gray-scale image $I: \mathbb{R}^{2} \rightarrow \mathbb{R}$ represented in the Beltrami framework by the mapping $X=(x, y, I)$. The first fundamental form is defined by:

$$
g_{\mu v}=\left(<\frac{\partial X}{\partial \mu}, \frac{\partial X}{\partial v}>\right)
$$

where we have here $\{\mu, v\}=\{x, y\}$, which implies:

$$
\begin{aligned}
& g_{x x}=1+I_{x}^{2}, \\
& g_{x y}=I_{x} I_{y}, \\
& g_{y y}=1+I_{y}^{2},
\end{aligned}
$$

where the suffixes stands for partial derivatives. We have:

$$
\frac{1}{\operatorname{det}\left(g_{\mu \nu}\right)}=\frac{1}{1+|\nabla I|^{2}}
$$

Function (2.1) corresponds (up to some constants) to the edge detector function used in the standard active contour model [10]. Hence, Sagiv et al. generalized the edge detector to texture images using the metric tensor of the Beltrami representation. Nevertheless, as we said earlier, the edge detector function has its limitations (sensitive to noise and initial contour position) and hence, it is not robust enough to segment a wide range of images. For this reason, Sagiv et al. coupled their edge detector with a region detector, coming from the vectorial Chan-Vese model [13], to segment complex textures. We observe that their segmentation model provides promising results, but the region-based part of the 
model (the vectorial Chan-Vese model) needs some computational time because it works with many channels. In order to improve the model of Sagiv et al. and decrease the computational time, we proposed in [27] to define an intrinsic region detector for textures in the Beltrami framework, rather than an edge detector. The proposed region detector for textures is computed once, and used in the active contour model. Thus, our active contour model did not need several channels, but only one feature image. This helped us to define a fast texture segmentation model in [27].

We used the shape operator and its eigenvalues to describe the geometry of textures. The shape operator is a linear operator which calculates the bending of a surface in different directions The eigenvalues of the shape operator correspond to the extremal of bending of the surface, they are called principal curvatures and they are known to represent the geometry of the considered smooth manifold. In [27], we observed that the simplest Beltrami representation of images, i.e. $X=(x, y, I)$, is enough to provide an efficient intrinsic texture feature to segment many natural images. In this Beltrami representation, the texture manifold is a 2-D manifold, with two unique principal curvatures $\left(\kappa_{1}, \kappa_{2}\right)$. The couple of principal curvatures $\left(\kappa_{1}, \kappa_{2}\right)$ defines an intrinsic descriptor for homogeneous textures. We made the assumption that for a given (complex) texture pattern, the couple $\left(\kappa_{1}, \kappa_{2}\right)$ follows the same distribution/pdf inside the texture region. This assumption is fulfilled in many natural images. Finally, the distribution of $\left(\kappa_{1}, \kappa_{2}\right)$ (or a combination of $\left.\kappa_{1}, \kappa_{2}\right)$ is automatically estimated through the segmentation process.

Based on $[25,34]$, the values of the principal curvatures in our particular mapping can be expressed as follows:

$$
\kappa_{1,2}=\frac{-\beta \pm \sqrt{\beta^{2}-4 \alpha \gamma}}{2 \alpha},
$$

where

$$
\left\{\begin{array}{l}
\alpha=1, \\
-\beta=\frac{1}{Z^{3}}\left[I_{x x}\left(1+I_{y}^{2}\right)+I_{y y}\left(1+I_{x}^{2}\right)-2 I_{x y}\left(I_{x} I_{y}\right)\right], \\
\gamma=\frac{1}{Z^{3}}\left[I_{x x} I_{y y}-\left(I_{x y}\right)^{2}\right] .
\end{array}\right.
$$

The first principal curvature $\kappa_{1}\left(\kappa_{1} \geq \kappa_{2}\right)$ corresponds to the maximal change of the normal to the surface and $\kappa_{2}$ corresponds to the minimal change. For sake of simplicity, and in order to use the information provided by the two principal curvatures, we considered to work with the norm of $\mathbf{k}_{1}+\mathbf{k}_{2}$, where vector $\mathbf{k}_{1}$ (resp. $\mathbf{k}_{2}$ ) has a norm $\kappa_{1}$ (resp. $\kappa_{2}$ ) and is oriented by the associated unit principal vector. Since $\mathbf{k}_{\mathbf{1}}$ and $\mathbf{k}_{2}$ are orthogonal, this leads to:

$$
\kappa_{t}:=\sqrt{\kappa_{1}^{2}+\kappa_{2}^{2}},
$$

where $\kappa_{t}: \mathbb{R}^{2} \rightarrow \mathbb{R}_{+}$defines the intrinsic texture descriptor that was used in [27] to segment real-world textures. The shape operator and its principal curvatures have proven to be efficient in [27] to segment a large class of textures since it outperforms state-of-the-art results. However, this texture descriptor is based on the computation of the second deriva- 
tive of $I$, which makes highly sensitive to noise. In the following section, we introduce a new texture descriptor based on semi-local image information, which is robust to noise.

\subsection{New texture feature based on semi-local image information}

In this section, we define an intrinsic texture descriptor, which is more robust and less sensitive to noise than the texture descriptor (2.2) used in [27]. To reach more robustness, we will change the local representation $X:(x, y) \rightarrow(x, y, I(x, y))$ into a semi-local representation. As we pointed out previously, textures are semi-local by nature, which motivates us to describe them with patches along the same line as non-local means s.a. $[9,21]$. Let $\mathscr{P}_{x, y}$ be the square patch of size $\tau \times \tau$ around the pixel $(x, y)$ :

$$
\mathscr{P}_{x, y}(I)=\left\{I\left(x+t_{x}, y+t_{y}\right)\right\}, \quad t_{x} \in\left[-\frac{\tau}{2}, \frac{\tau}{2}\right], \quad t_{y} \in\left[-\frac{\tau}{2}, \frac{\tau}{2}\right] .
$$

We consider the new representation of textures in the Beltrami framework with the following mapping:

$$
X:(x, y) \rightarrow\left(X_{1}=x, X_{2}=y, X_{3}=\mathscr{P}_{x, y}(I)\right) .
$$

This mapping includes local information (position in space) and semi-local image information (the patch with the neighborhood pixel values around the current pixel). We make the assumption that for a given (complex) texture pattern, the geometry of the manifold embedded by the mapping (2.4) will be uniform for the observed texture. This assumption is actually fulfilled in many natural images. This implies that the metric tensor of the manifold is actually homogeneous in regions with same texture. We will use this assumption to define a new intrinsic texture descriptor, more robust than the one introduced in [27] and in the previous section. A justification of this observation comes from the definition of the metric tensor, which measures distances between points on the manifold. If the manifold is almost flat in a certain region of the manifold, then the distance between two points anywhere on this region is almost equal. The manifold, represented by (2.4), is almost flat in regions with same textures because of the semi-local image information. The corresponding metric tensor of (2.4) is as follows:

$$
g_{x y}=\left(\begin{array}{cc}
1+\left(\partial_{x} \mathscr{P}_{x, y}\right)^{2} & \partial_{x} \mathscr{P}_{x, y} \partial_{y} \mathscr{P}_{x, y} \\
\partial_{x} \mathscr{P}_{x, y} \partial_{y} \mathscr{P}_{x, y} & 1+\left(\partial_{y} \mathscr{P}_{x, y}\right)^{2}
\end{array}\right),
$$

Finally, the intrinsic texture descriptor, namely $F$, is defined as:

$$
F=\exp \left(-\frac{\operatorname{det}\left(g_{x y}\right)}{\sigma^{2}}\right)
$$

where $\sigma>0$ is a scaling parameter. The use of the Gaussian kernel acts as a low-pass filter, which controls the degree of details. Fig. 1 summarizes the whole process.

For vector-valued images s.a. color images, the extension is straightforward. Let $\mathbf{I}=$ $\left(I_{1}, I_{2}, \cdots, I_{k}\right)$ be a vector-valued image, where $k$ is the number of channels. Then, the corresponded semi-local mapping is:

$$
X:(x, y) \rightarrow\left(X_{1}=x, X_{2}=y, X_{3}=\mathscr{P}_{x, y}\left(I_{1}\right), \cdots, X_{2+k}=\mathscr{P}_{x, y}\left(I_{k}\right)\right) .
$$




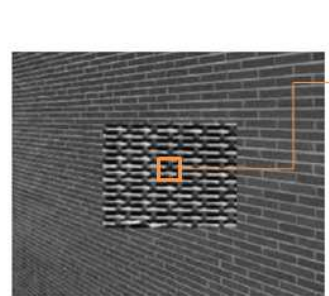

Original Image

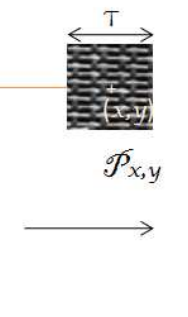

Beltrami representation based on semi-local image information

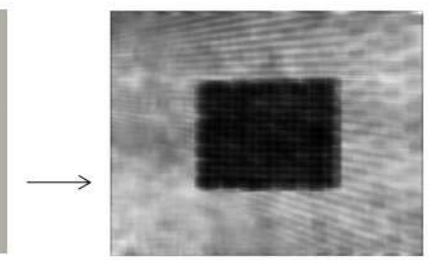

Intrinsic texture descriptor

Figure 1: Illustration of the semi-local representation and result of the texture feature.

and the metric tensor has the following form:

$$
g_{x y}=\left(\begin{array}{cc}
1+\sum_{j=1}^{k}\left(\partial_{x} \mathscr{P}_{x, y}\right)\left(I_{j}\right)^{2} & \sum_{j=1}^{k}\left(\partial_{x} \partial_{x} \mathscr{P}_{x, y}\left(I_{j}\right) \partial_{y} \mathscr{P}_{x, y}\left(I_{j}\right)\right. \\
\sum_{j=1}^{k} \partial_{x} \mathscr{P}_{x, y}\left(I_{j}\right) \partial_{y} \mathscr{P}_{x, y}\left(I_{j}\right) & 1+\sum_{j=1}^{k}\left(\partial_{y} \mathscr{P}_{x, y}\left(I_{j}\right)\right)^{2}
\end{array}\right),
$$

In our experiments, we will segment color images represented in the three primary color space: red, green, blue (RGB), see Section 5 .

\section{Unsupervised active contour model based on the Kullback-Leibler distance}

In this section, we introduce an unsupervised segmentation model based on the active contour model and the Kullback-Leibler (KL) Distance between histograms/probability density functions (pdfs). We show that the segmentation model is mathematically wellposed since a minimizing solution exists in the space of functions with bounded variation.

\subsection{Proposed segmentation method}

We propose to solve the unsupervised image segmentation problem for gray-scale images composed of an object and the background (two-phase segmentation). An efficient way to perform an unsupervised segmentation task is to use the Region Competition approach introduced by Zhu and Yuille in [56]. We will perform a histogram/pdf competition approach based on the KL distance, which measures the distance between two pdfs. This segmentation model has been introduced in [27] and has shown promising results. The method consists in maximizing the $\mathrm{KL}$ distance between the pdf inside and outside the evolving contour, which defines two regions representing the object of interest and the background.

In what follows, we describe the method with the intrinsic texture descriptor $F: \mathbb{R}^{2} \rightarrow$ $\mathbb{R}_{+}$introduced in (2.5). However, the function $F$ can be replaced by any scalar feature function such as the gray-level value $I$ of the image or the texture feature based on the shape operator as done in [27]. Let $q_{\text {in }}$ be the inside pdf, $q_{\text {out }}$ the outside one, $\Omega=\Omega_{\text {in }}$ be the evolving region and $\Omega_{0} \backslash \Omega=\Omega_{\text {out }}$ it complementary in the image domain $\Omega_{0}$. In this approach, the texture feature $F$ is considered as a random variable. The set of possible 
outcomes is $\mathbb{R}_{+}$and the pdfs $q_{\text {in }}$ and $q_{\text {out }}$ associated with an observation $F$ for a given region $\Omega$ at a fixed moment are defined by:

$$
\left\{\begin{array}{l}
q_{\text {in }}(F, \Omega)=\frac{1}{|\Omega|} \int_{\Omega} K(F-F(\hat{x})) d \hat{x}, \\
q_{\text {out }}(F, \Omega)=\frac{1}{\left|\Omega_{0} \backslash \Omega\right|} \int_{\Omega_{0} \backslash \Omega} K(F-F(\hat{x})) d \hat{x},
\end{array}\right.
$$

where |.| is the area of the given region. The new KL distance is thus as follows:

$$
\begin{aligned}
& K L\left(q_{\text {in }}(\Omega), q_{\text {out }}(\Omega)\right) \\
= & \left.\int_{\mathbb{R}_{+}}\left(q_{\text {in }}(F, \Omega) \frac{q_{\text {in }}(F, \Omega)}{q_{\text {out }}(F, \Omega)}\right)+q_{\text {out }}(F, \Omega) \log \frac{q_{\text {out }}(F, \Omega)}{q_{\text {in }}(F, \Omega)}\right) d F \\
= & \int_{\mathbb{R}_{+}}\left(q_{\text {in }}(F, \Omega)-q_{\text {out }}(F, \Omega)\right) \cdot\left(\log q_{\text {out }}(F, \Omega)-\log q_{\text {in }}(F, \Omega)\right) d F .
\end{aligned}
$$

Functional (3.1) gives a measure of difference between the pdfs defined inside and outside, for a fixed active contour represented by $\Omega$. One naturally wants to maximize Functional (3.1) in order to determine two regions with two pdfs as disjoint as possible, representing the object of interest and the background. Maximizing Functional (3.1) involves the computation of its derivatives w.r.t the evolving domain $\Omega$, which can be done with the shape derivative tool $[1,29]$. Using the shape derivative tool, the Eulerian derivative in the direction $\mathbf{V}$ of the criterion (3.1) is as follows (see Annex A.1):

$$
\begin{aligned}
& <K L^{\prime}, \mathbf{V}> \\
= & \int_{\partial \Omega}\left\{\frac{1}{|\Omega|} \int_{\mathbb{R}_{+}}\left[1-\frac{q_{\text {out }}(F, \Omega)}{q_{\text {in }}(F, \Omega)}+\log \frac{q_{\text {in }}(F, \Omega)}{q_{\text {out }}(F, \Omega)}\right] \cdot\left[-K(F-F(s))+q_{\text {in }}(F, \Omega)\right] d F\right. \\
& \left.-\int_{\mathbb{R}_{+}} \frac{1}{\left|\Omega_{0} \backslash \Omega\right|}\left[1-\frac{q_{\text {in }}(F, \Omega)}{q_{\text {out }}(F, \Omega)}+\log \frac{q_{\text {out }}(F, \Omega)}{q_{\text {in }}(F, \Omega)}\right] \cdot\left[-K(F-F(s))+q_{\text {out }}(F, \Omega)\right] d F\right\} \\
& <\mathrm{V}(s) \cdot \mathscr{N}(s)>d s,
\end{aligned}
$$

where $\mathscr{N}$ is the unit inward normal to $\partial \Omega$ the boundary of the evolving region $\Omega, d s$ its length/area element. According to the Cauchy-Schwartz inequality, the fastest way to decrease energy $K L(\Omega(\tau))$ is obtained by choosing $\frac{\partial C}{\partial \tau}=-\mathbf{V} . \mathscr{N}$, where $\tau$ is an artificial time and $C=\partial \Omega$, which leads to the evolution equation:

$$
\begin{aligned}
\frac{\partial C}{\partial \tau}= & \left\{\int_{\mathbb{R}_{+}} \frac{1}{|\Omega|}\left[1-\frac{q_{\text {out }}(F, \Omega)}{q_{\text {in }}(F, \Omega)}+\log \frac{q_{\text {in }}(F, \Omega)}{q_{\text {out }}(F, \Omega)}\right] \cdot\left[K(F-F(s))-q_{\text {in }}(F, \Omega)\right] d F\right. \\
& +\int_{\mathbb{R}_{+}} \frac{1}{\left|\Omega_{0} \backslash \Omega\right|}\left[1-\frac{q_{\text {in }}(F, \Omega)}{q_{\text {out }}(F, \Omega)}+\log \frac{q_{\text {out }}(F, \Omega)}{q_{\text {in }}(F, \Omega)}\right] \\
& \left.\cdot\left[-K(F-F(s))+q_{\text {out }}(F, \Omega)\right] d F+\lambda \kappa\right\} \mathscr{N}
\end{aligned}
$$


where the last term $\lambda \kappa, \lambda>0$ has been added in the evolution equation in order to regularize the evolving curve. $\kappa$ is the curvature of the contour $C$ and it is derived from the minimization of the curve length $\int_{\partial \Omega} d s$.

\subsection{Existence of a minimizing solution for the proposed model (3.1)}

In this section, we show the existence of a minimizer of the variational problem (3.1) associated with the length term by the standard method of calculus of variations and the space of functions with bounded variation. Our energy Functional is composed of two terms: the first one is the Kullback-Leibler functional and the second term is a curvature regularization term.

We assume that the function $F$ is a function $F \in L^{\infty}\left(\Omega_{0}\right)$, where $\Omega_{0}$ is a regular open bounded set of $\mathrm{R}^{n}$ corresponding to the image domain. We define the set $\mathscr{U}$ of all image regions in $\Omega_{0}$, i.e. the set of regular open bounded sets of $\Omega_{0}$.

The image segmentation problem proposed in (3.1) consists in finding a set $\Omega \in \mathscr{U}$ which minimizes the following functional:

$$
E(\Omega)=\underbrace{\int_{\mathbb{R}_{+}}\left(q_{\text {in }}(F, \Omega) \log \frac{q_{\text {in }}(F, \Omega)}{q_{\text {out }}(I, \Omega)}+q_{\text {out }}(F, \Omega) \log \frac{q_{\text {out }}(F, \Omega)}{q_{\text {in }}(F, \Omega)}\right)}_{K L(\Omega)}+\lambda \underbrace{\int_{\partial \Omega} d s}_{L(\Omega)}
$$

where the non-parametric pdfs $q_{\text {in }}$ and $q_{\text {out }}$ are estimated with a Parzen estimation [43] as follows:

$$
\left\{\begin{array}{l}
q_{\text {in }}(F, \Omega)=\frac{1}{|\Omega|} \int_{\Omega} K(F-F(\hat{x})) d \hat{x}, \\
q_{\text {out }}(F, \Omega)=\frac{1}{\left|\Omega_{0} \backslash \Omega\right|} \int_{\Omega_{0} \backslash \Omega} K(F-F(\hat{x})) d \hat{x},
\end{array}\right.
$$

where $\Omega_{0} \backslash \Omega$ is the complement set of $\Omega$ in $\Omega_{0}$.

We look for a set $\Omega \in \mathscr{U}$ which minimizes the region functional (3.4). As it is pointed out in $[1,29]$, the optimization of the functional (3.4) is difficult to carry out since the set $\mathscr{U}$ of regular domains does not have the structure of a vector space. The variation of the domain is thus done through a family of homeomorphism transformations $T$ (i.e. one-to-one with $T$ and $T^{-1}$ continuous), which allows to differentiate $E$ with respect to $\Omega$ and determine a minimization flow in Section 3.1. Thus, one possible approach to prove the existence of minimizers for (3.4) is to express (3.4) in term of the transformation $T$ and look for a minimizer $T_{\star}$. However, we decide to choose another approach to prove the existence of a minimizer which looks easier. This approach consists, in a first step in re-writing the functional $F$ with the characteristic function $\chi_{\Omega}$ of the set $\Omega$, defined as follows:

$$
\chi_{\Omega}(x)= \begin{cases}1 & \text { if } x \in \Omega \in \mathscr{U} \\ 0 & \text { otherwise }\end{cases}
$$


and then applying the standard method of the calculus of variations to prove the existence of a minimizer. Functional (3.4) can be expressed w.r.t. $\chi_{\Omega}$ :

$$
F\left(\chi_{\Omega}\right)=K L\left(\chi_{\Omega}\right)+\lambda L\left(\chi_{\Omega}\right)
$$

where

$$
L\left(\chi_{\Omega}\right)=\int_{\Omega_{0}}\left|\nabla \chi_{\Omega}\right| d x
$$

and

$$
\left\{\begin{array}{l}
q_{\text {in }}\left(F, \chi_{\Omega}\right)=\frac{\int_{\Omega_{0}} K(F-F(\hat{x})) \chi_{\Omega}(\hat{x}) d \hat{x}}{\int_{\Omega_{0}} \chi_{\Omega} d \hat{x}}, \\
q_{\text {out }}\left(F, \chi_{\Omega}\right)=\frac{\int_{\Omega_{0}} K(F-F(\hat{x}))\left(1-\chi_{\Omega}(\hat{x})\right) d \hat{x}}{\int_{\Omega_{0}}\left(1-\chi_{\Omega}\right) d \hat{x}} .
\end{array}\right.
$$

(3.6) is the total variation (TV) norm, which will play an important role in the theorem of existence. It is defined according to:

Definition 3.1 ([19,23]). Let $\Omega \in \mathscr{U}$ and $u \in L^{1}\left(\Omega_{0}\right)$. The TV norm of the function $u$ is defined as follows:

$$
T V(u)=\int_{\Omega_{0}}|\nabla u| d x=\sup _{\phi \in \Phi}\left\{\int_{\Omega_{0}} u \operatorname{div} \phi d x\right\},
$$

where $\Phi=\left\{\phi \in C_{0}^{1}\left(\Omega_{0}, \mathbb{R}^{n}\right)|| \phi \mid \leq 1\right.$, on $\left.\Omega_{0}\right\}$ and $C_{0}^{1}\left(\Omega_{0}, \mathbb{R}^{n}\right)$ are the continuously differentiable real functions on $\Omega_{0}$.

Moreover,

Definition 3.2 ([19,23]). A function $u \in L^{1}\left(\Omega_{0}\right)$ is said to have bounded variation in $\Omega_{0}$ if its distributional derivative satisfies $T V(u)<\infty$. We define $B V\left(\Omega_{0}\right)$ as the space of all functions in $L^{1}\left(\Omega_{0}\right)$ with bounded variation. The space $B V\left(\Omega_{0}\right)$ is a Banach space, endowed with the norm:

$$
\|u\|_{B V\left(\Omega_{0}\right)}=\|u\|_{L^{1}\left(\Omega_{0}\right)}+T V(u) .
$$

We introduce two important theorems that is already used in Eq. (3.6) and will be used in our theorem of existence.

Theorem 3.1 ([19,23]). A set $\Omega \in \mathscr{U}$ has finite perimeter if and only if the characteristic function $\chi_{\Omega}$ of $\Omega$ belongs to $B V\left(\Omega_{0}\right)$. We have

$$
\operatorname{Per}(\Omega)=T V\left(\chi_{\Omega}\right)=\int_{\Omega_{0}}\left|\nabla \chi_{\Omega}\right| d x<\infty,
$$


and

Theorem 3.2 ([19,23]). Let $\Omega \in \mathscr{U}$. If $\left\{u_{k}\right\}_{k \geq 1}$ is a bounded sequence in $B V\left(\Omega_{0}\right)$, then there exists a subsequence $\left\{u_{k_{j}}\right\}$ of $\left\{u_{k}\right\}$ and a function $u_{\star} \in B V\left(\Omega_{0}\right)$, such that $u_{k_{j}} \rightarrow u_{\star}$ strongly in $L^{p}\left(\Omega_{0}\right)$ for any $1 \leq p<n /(n-1)$ and

$$
T V\left(u_{\star}\right) \leq \liminf _{k_{j} \rightarrow \infty} T V\left(u_{k_{j}}\right) .
$$

We can now state the theorem of the existence of (at least) one minimizer for (3.5):

Theorem 3.3. Our minimization problem

$$
\min _{\chi_{\Omega} \in B V\left(\Omega_{0}\right)}\left\{K L\left(\chi_{\Omega}\right)+\lambda L\left(\chi_{\Omega}\right)\right\}, \lambda>0
$$

has a solution in $B V\left(\Omega_{0}\right)$.

Proof. The direct method of the calculus of variations $[2,15,52]$ is used:

A) Let $\left\{\chi_{\Omega_{k}}\right\}_{k \geq 1}$ be a minimizing sequence of (3.8), i.e.

$$
\lim _{k \rightarrow \infty} F\left(\chi_{\Omega_{k}}\right)=\inf _{\chi_{\Omega} \in B V\left(\Omega_{0}\right)} F\left(\chi_{\Omega}\right)
$$

B) Since $\chi_{\Omega_{k}}$ is a sequence of characteristic functions of the sets $\Omega_{k}$, then $\chi_{\Omega_{k}}(x) \in$ $\{0,1\}$ - a.e. in $\Omega_{0}$. A constant $M>0$ exists such that $\left\|\nabla \chi_{\Omega_{k}}\right\|_{L^{1}\left(\Omega_{0}\right)} \leq M, \forall k \geq 1$. Therefore, $\chi_{\Omega_{k}}$ is a uniformly bounded sequence on $B V\left(\Omega_{0}\right)$. Following Theorem 3.2, a subsequence of $\chi_{\Omega_{k_{j}}}$ that converges to a function $\chi_{\Omega_{\star}}$ strongly in $L^{1}\left(\Omega_{0}\right)$ exists.

C) Taking a minimizing sequence $\chi_{\Omega_{k_{j}}} \rightarrow \chi_{\Omega_{\star}}$, it is easy to show that $\lim _{k_{j} \rightarrow \infty} K L\left(\chi_{\Omega_{k_{j}}}\right)=$ $K L\left(\chi_{\Omega_{\star}}\right)$ since $q_{\text {in }}\left(\chi_{\Omega}\right)$ and $q_{\text {out }}\left(\chi_{\Omega}\right)$ in Eq. (3.7) are continuous w.r.t. the $B V\left(\Omega_{0}\right)$ topology. Thus, according to Theorem 3.2, we deduce that

$$
F\left(\chi_{\Omega_{\star}}\right) \leq \liminf _{k_{j} \rightarrow \infty} F\left(\chi_{\Omega_{k_{j}}}\right)
$$

which implies that

$$
F\left(\chi_{\Omega_{\star}}\right)=\min _{\chi_{\Omega} \in B V\left(\Omega_{0}\right)} F\left(\chi_{\Omega}\right)
$$

which means that $\chi_{\Omega_{\star}}$ is a minimizer of $F$ among characteristic functions $\chi_{\Omega}$ of sets $\Omega \in \mathscr{U}$ of finite perimeter in $\Omega_{0}$. It also implies the existence of at least one set $\Omega_{\star}$, given by $\left\{x \in \Omega_{0} \mid \chi_{\Omega_{\star}}(x)=1\right\}$, which minimizes (3.4). 


\section{Convexification and fast minimization algorithm}

\subsection{Convexification of the proposed energy functional}

The standard approach to minimize energy functional such as (3.1) (associated with the length term) with active contour is using the Level Set Method (LSM) introduced by Osher and Sethian in [40]. However, the LSM is a slow minimization technique that only determines a local minimum of the energy functional. The authors in $[5,12]$ introduced a new paradigm to compute a global minimizer of the active contour energy in a fast way. More precisely they proposed an equivalent convex formulation of energy (3.1) and then used fast minimization schemes to reach the optimal solution.

We apply this paradigm in this section to quickly minimize (3.1).

The LSM applied to the minimization of energy (3.1) produces the following PDE:

$$
\frac{\partial \phi}{\partial \tau}=\left(-\lambda V_{K L}+\nabla \frac{\nabla \phi}{|\nabla \phi|}\right)|\nabla \phi|
$$

where $V_{K L}$ is the speed provided by Kullback-Leibler distance (3.1). We assume that $V_{K L}$ is fixed. Since $|\nabla \phi|>0$, the steady state of (4.1) is the same as:

$$
\frac{\partial \phi}{\partial \tau}=-\lambda V_{K L}+\nabla \frac{\nabla \phi}{|\nabla \phi|} .
$$

The variational model that provides (4.2) is also given by:

$$
\min _{\phi \in[0,1]} \int_{\Omega_{0}}-\lambda V_{K L} \phi+|\nabla \phi| .
$$

Finally, we change the notation of the function $\phi$ to avoid any confusion with the LSM. We will seek the minimum of the convex functional $F(u)$ defined by:

$$
\min _{u \in[0,1]} \int_{\Omega_{0}}-\lambda V_{K L} u+|\nabla u|,
$$

where $\int_{\Omega_{0}}|\nabla u|=T V(u)$ is the total variation norm of the function $u$.

\subsection{Fast minimization algorithm based on the Split-Bregman method}

In [27], we introduced a minimizing algorithm based on [5]. Although the algorithm provides relatively fast minimization, it has some limitations. First, the algorithm of $[5,27]$ is based on a splitting/regularization approach (along the same lines as [3]) to minimize (4.4). In [27], the authors minimized:

$$
\min _{u, v \in[0,1]} F(u, v)=\int_{\Omega_{0}}-\lambda V_{K L} v+|\nabla u|+\frac{1}{2 \theta}(u-v)^{2},
$$


where the right-most term enforces $u \approx v$ for sufficiently small $\theta$. Minimization with respect to $u$ corresponds to solving the ROF model [46], which is done using a gradient projection method [11], and the minimization for $v$ can be solved using an explicit formula. However, this scheme slows down as the accuracy increases (i.e., as $\theta \rightarrow 0$ ). Second, the approximate enforcement of the constraint $u \approx v$ has the effect of regularizing the model. The disadvantage of these regularized schemes is that they "smear" the values of $u$ near the objects boundaries. This makes the results more dependent on the value of the cutoff parameter and can eliminate fine segmentation details. In [24], the authors use the Split-Bregman method to overcome regularization effect. Besides, the proposed method of [24] has the advantage of being a much more efficient solver for (4.4) since the Projection's algorithm has a linear convergence property whereas experiments show that the Split-Bregman has a quadratic convergence property (it was shown in [24] that the Split-Bregman method is actually slightly faster than graph-cuts method). The first step consists in introducing a new variable $d$ such that the new system to solve becomes:

$$
\min _{u, d} \int_{\Omega_{0}}-\lambda V_{K L} u+|d|, \quad \text { s.t. } d=\nabla u .
$$

We apply the Split-Bregman iterative scheme to minimize (4.5) (see [24] for more details) which leads to the following unconstrained problem:

$$
\left\{\begin{array}{l}
\left(u^{k+1}, d^{k+1}\right)=\arg \min _{0 \leq u \leq 1, d}\|d\|+\lambda V_{K L} u+\frac{\beta}{2}\left\|d-\nabla u-b^{k}\right\|_{2}^{2}, \\
b^{k+1}=b^{k}+\nabla u^{k+1}-d^{k+1}
\end{array}\right.
$$

starting from $b^{k=0}=d^{k=0}=u^{k=0}=0$. The minimizing solution w.r.t. $u$ in (4.6) holds the optimality condition:

$$
\begin{aligned}
& 0=\lambda V_{K L}+\frac{\beta}{2} \operatorname{div}\left(d^{k}-\nabla u-b^{k}\right), \\
& \beta \triangle u=\lambda V_{K L}+\beta \operatorname{div}\left(d^{k}-b^{k}\right) .
\end{aligned}
$$

An approximate solution is given by a Gauss-Seidel iterative method, $n \geq 0$ :

$$
\begin{aligned}
& \gamma_{i, j}=d_{i-1, j}^{x, k}-d_{i, j}^{x, k}-b_{i-1, j}^{x, k}+b_{i, j}^{x, k}+d_{i, j-1}^{y, k}-d_{i, j}^{y, k}-b_{i, j-1}^{y, k}+b_{i, j}^{y, k}, \\
& \mu_{i, j}=\frac{1}{4}\left(u_{i-1, j}^{k, n}+u_{i+1, j}^{k, n}+u_{i, j+1}^{k, n}+u_{i, j+1}^{k, n}-\frac{\lambda}{\beta} V_{K L}+\gamma_{i, j}\right), \\
& u_{i, j}^{k+1, n+1}=\max \left\{\min \left\{\mu_{i, j}, 1\right\}, 0\right\} .
\end{aligned}
$$

Finally, the solution of (4.6) w.r.t. $d$ is given by a soft-wavelet thresholding s.t.:

$$
d^{k+1}=\operatorname{sign}\left(\nabla u^{k+1}+b^{k}\right) \max \left(\left|\nabla u^{k+1}+b^{k}\right|-\lambda^{-1}, 0\right) .
$$




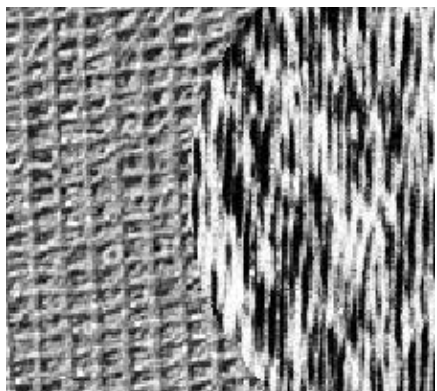

(a)

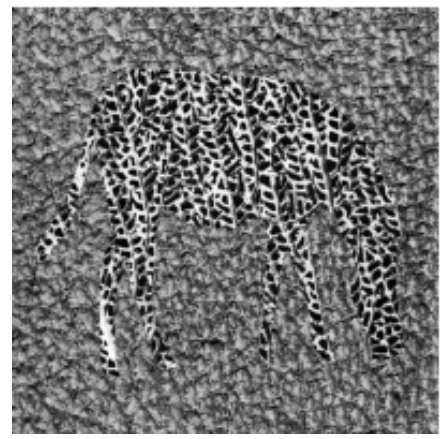

(d)

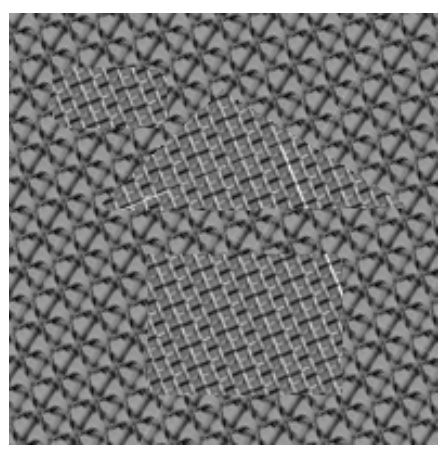

(g)

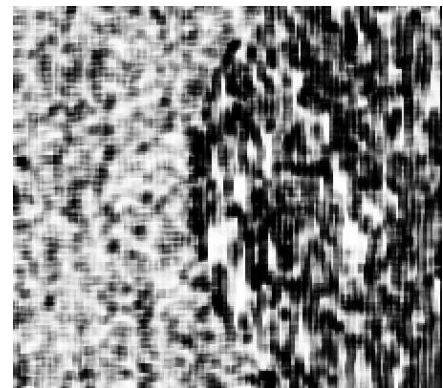

(b)

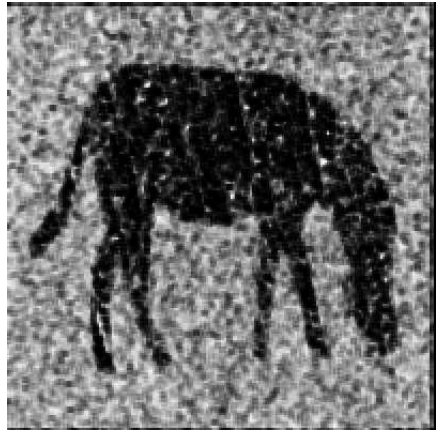

(e)

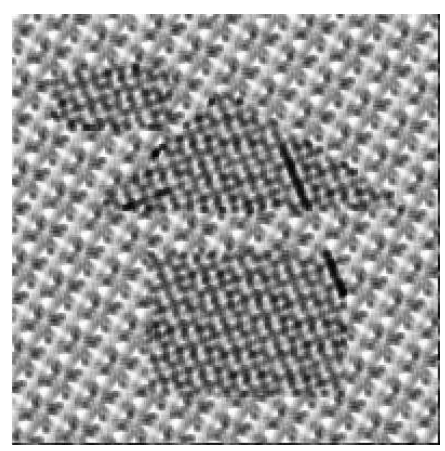

(h)

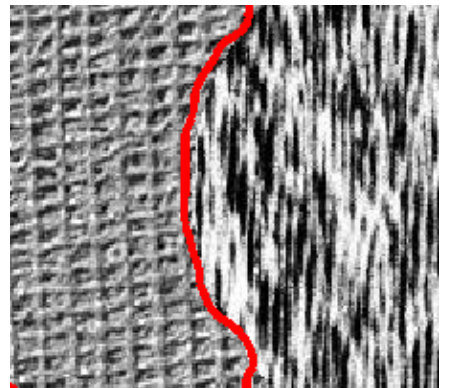

(c)

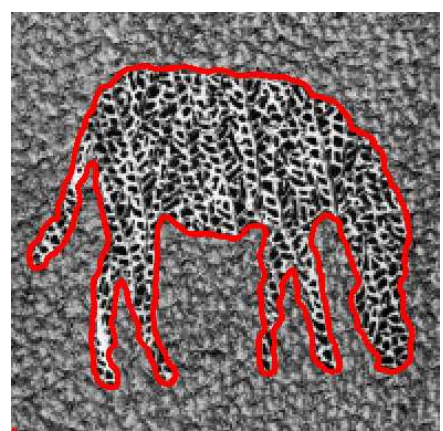

(f)

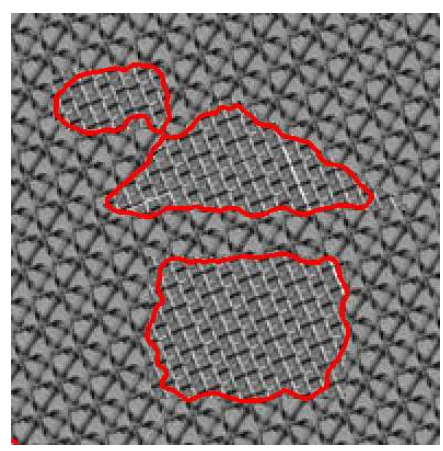

(i)

Figure 2: Segmentation of synthetic images. Left column: original images. Center column: Result of the intrinsic texture descriptor. Right column: our segmentation result.

\section{Experimental results}

We applied our texture segmentation algorithm to a set of challenging synthetic and real-world textural images. The synthetic textural images, Figs. 2(a), 2(d), 2(g) were generated from the Brodatz data set [7]. The natural textural images, Figs. 3(a), 3(d), $3(\mathrm{~g}), 3(\mathrm{j}), 3(\mathrm{~m})$ were taken in the Berkeley data set [39].

The first step is to extract homogeneous regions from the textured images following the method proposed in Section 2.2. Figs. 2(b), 2(e), 2(h) show the results of the in- 


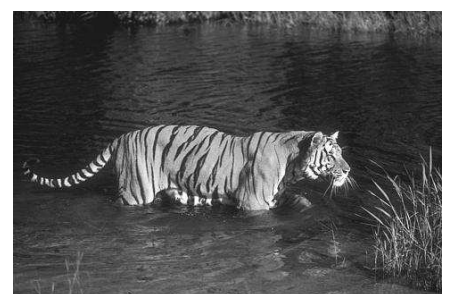

(a)

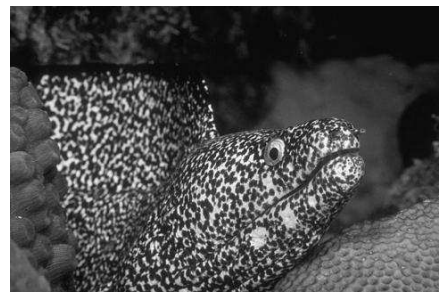

(d)

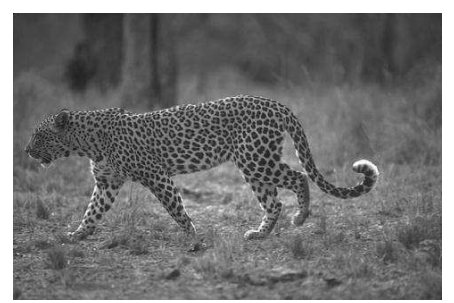

(g)

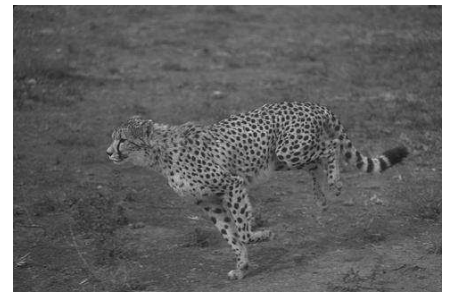

(j)

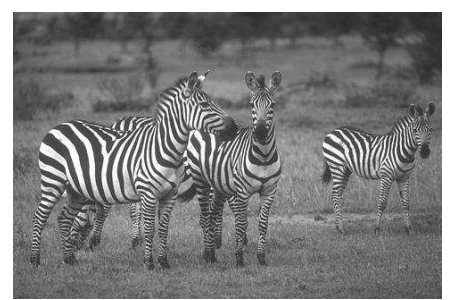

(m)

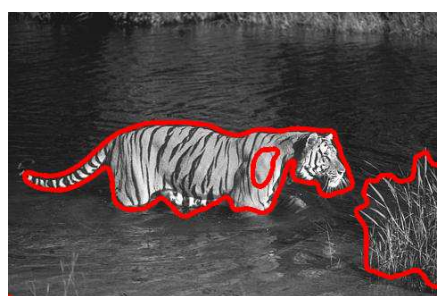

(b)

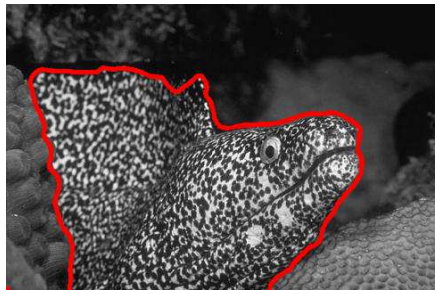

(e)

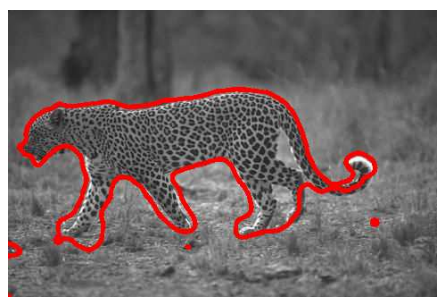

(h)

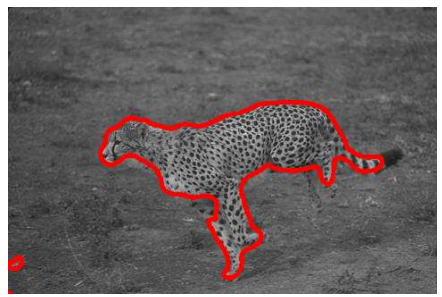

(k)

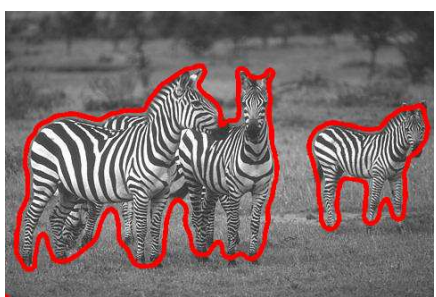

(n)

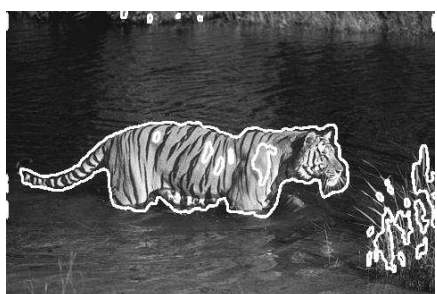

(c)

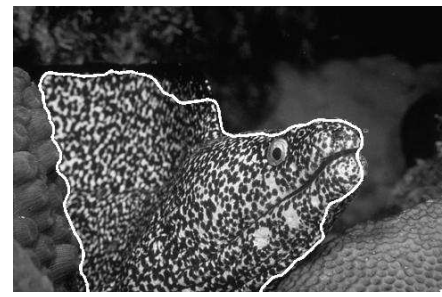

(f)

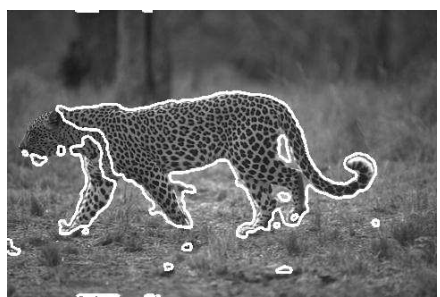

(i)

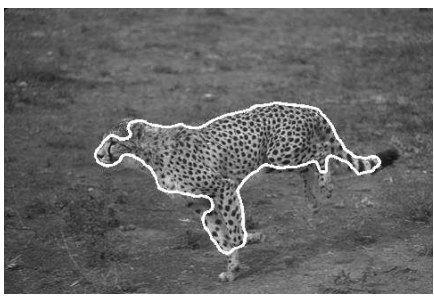

(l)

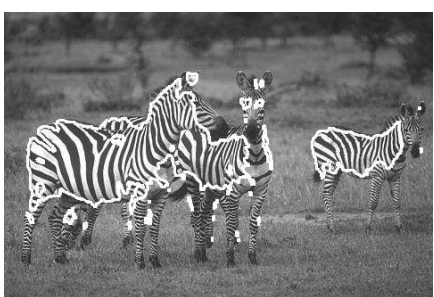

(o)

Figure 3: Segmentation of real-world textural images. Left column: original images. Center column: our segmentation result. Right column: results based on the method [47]. 
trinsic texture descriptor applied to the synthetic textures. We observed that the images of the intrinsic texture descriptor are more "homogeneous" than the original images, and one would like to apply the well-known Chan-Vese model [14] to segment the textures. However, although the Chan-Vese model can segment some textures such as the textured zebra, it will fail for more complicated textures. The intrinsic texture feature is much better represented by a probability density function, as we proposed in our segmentation model.

The quality of the texture descriptor depends on the patch size. The patch should be large enough to contain enough discriminative information between the object and the background. However with a larger patch we note a loss of accuracy in the borders. The unsupervised segmentation process proposed in Section 3 is then applied on the texture feature and the fast minimization scheme based on the Split-Bregman algorithm is used (Section 4.2).

Fig. 2, right column, presents the results obtained for synthetics texture. Our model has managed to distinguish the different texture classes and can deal with multiple object and non-convex shapes.

As a comparison with the state-of-the-art techniques, we decided to implement the efficient texture segmentation model of Savig et al. [47], which uses the vectorial ChanVese model [13] and an edge detector function based on Gabor responses as explained in Section 2.1. We modified their original model by implementing a dual formulation of their energy functional as done in [5]. Besides, the selected Gabor features are chosen with a simple selection criteria defined in [28] in order to have the most relevant collection of Gabor features. Fig. 3 presents the results obtained with our method on the center column and the model of Sagiv et al. in the right column. Our method manages to capture with more regularity and precision the object. We notice that in the case of the tiger image (Fig. 3(a), the fact that some part (part larger than the size patch) are smooth and not textured mislead the segmentation. For the images with more regular textures, the segmentation is satisfactory.

Finally we applied our algorithm to color images (Fig. 4). Results are shown in the right column.

We notice that our segmentation model needs to choose three parameters: $\theta, \lambda$ as explained in Section 4.2 and $\sigma$ for the Parzen estimation in Section 3.1. In all experiments, the mean computing time for both the feature extraction and the segmentation is around 10 seconds for an image $481 \times 321$ (including the updated estimation of the pdfs), whereas, for the same image, the texture segmentation model in [47] adapted to the fast minimization scheme in [5] takes around 4 minutes.

\section{Discussion and conclusion}

In this paper, we have proposed an unsupervised segmentation method for a large class of synthetic and natural textures. Several aspects of this difficult problem have been considered and lead us to use different theories and tools.

The first step in the segmentation process is to define and extract texture features. This is an important and common step for the texture segmentation task. For instance in [44], 


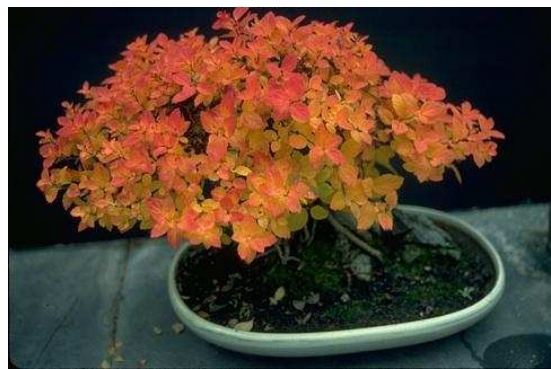

(a)

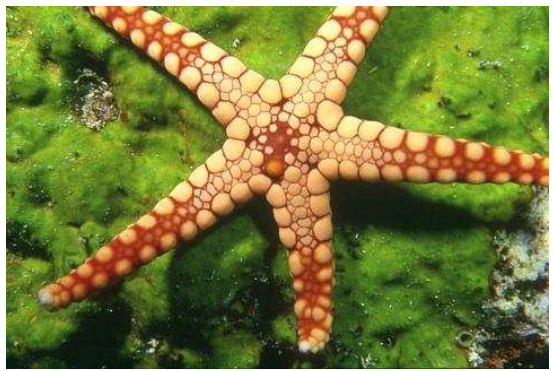

(c)

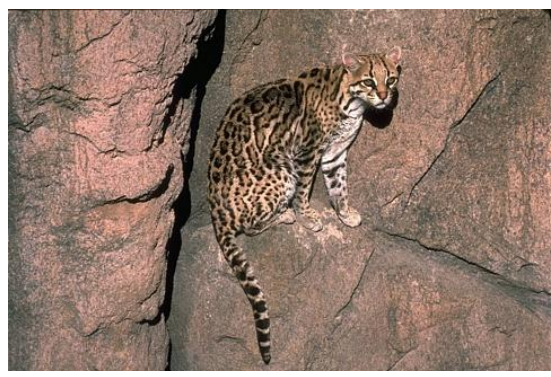

(e)

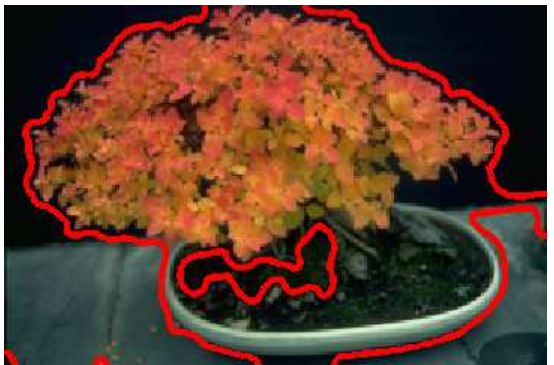

(b)

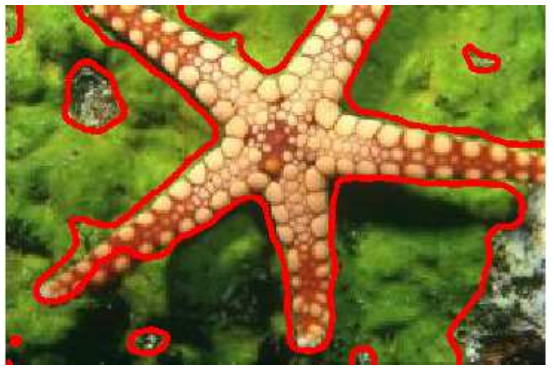

(d)

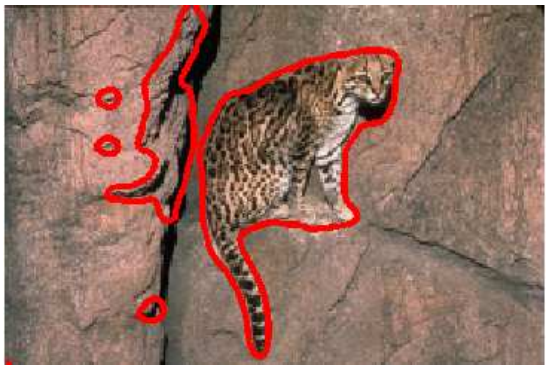

(f)

Figure 4: Segmentation of real-world color images. Left column: original images. Right column: our segmentation result.

Rousson et al. used a non-linear diffusion of the structure tensor from which the statistical information was extracted. Although this method provides interesting segmentation results, it is limited for highly disordered textures or complex natural images. Besides, this method requires working with $N=5$ image features, unlike our model which requires one image feature. Other texture segmentation algorithms have been developed on Gabor filters. In fact, Gabor filters are much appreciated for texture segmentation because these filters can extract complex geometry from images. However, methods based on Gabor filters can be time consuming because they require using many image features which sometimes do not hold much useful information, see for example [13]. The proposed texture descriptor developed in this paper shows a good quality to represent complex and natural textures. The proposed texture descriptor has been developed from the Beltrami 
framework and semi-local image information. The Beltrami framework offers a natural approach to define a high-dimensional manifold from the image patches and allows to define a texture descriptor based on the intrinsic geometry of this geometrical representation. The proposed texture feature has provided promising results to segment complex textures with different orientations and scales. We believe that the patch information, which represents semi-local information, is a natural and efficient way to extract texture information. From a numerical point of view, the proposed texture descriptor is easy to implement and an image of size $256 \times 256$ can be processed in few seconds. Besides, the proposed texture descriptor does not use several channels, like in the case of Gabor filters, but only one function, which makes possible fast texture segmentation. The limitation of this texture descriptor lies in the patches size. In our implementation, the size is fixed and is the same for all points in the image. However, the size of textures can change in the image. A solution could be to increase the size of patches but we observed that larger sizes produce less precision in the segmentation process. We will investigate how to change the size of patches to match the different textures lying in the images.

In the texture segmentation task, the feature extraction step is followed by the segmentation process, which produces the optimal two-phase partition of the image. In this paper, we were motivated to have a totally user-free algorithm, i.e. an unsupervised segmentation algorithm. We chose to maximize the Kullback-Leibler (KL) distance between the probability density function (pdf) of the object of interest and the pdf of the background. The maximum of the KL produces two regions with the pdfs as disjoint as possible. The $\mathrm{KL}$ distance has proven to be a good distance measure between two pdfs, despite a certain computational cost. This distance, borrowed from Information Theory, has good theoretical properties such as invariance w.r.t. illumination changes, which is an important property to segment natural images. The KL distance has proven to be an effective tool for other applications. For instance, Freedman and Zhang [20] used the KL measure for object tracking. Based on a density probability model, the KL flow allows the matching between the model and the distribution of the current region in the image. Another application is given by Wang and Vemuri in [53] for Diffusion Tensor-MRI data, where the authors redefined the $\mathrm{KL}$ measure for a positive definite $2 \mathrm{~d}$-tensor. The segmentation process is done by minimizing the KL divergence between an inside average tensor and tensors inside the active contour and an outside average tensor and the tensors outside de active contour. In contrast with other segmentation methods based on comparison of pdfs such as [30,42], we do not use any prior assumptions on the probability distributions. In [42], Paragios and Deriche based their segmentation process on the Gaussian distribution hypothesis of the regions to be segmented. In [30], Jehan-Besson et al. used a prior histogram of the region of interest to perform the segmentation. Our segmentation model, based on a region competition approach, performs an unsupervised segmentation task. The unsupervised segmentation is done through an active contour model, which maximizes the KL distance while regularizing the contour. Active contour models are known to compute a local minimizer of the energy functional and these models are slow numerically. However, we have developed a fast numerical minimization algorithm, based on the Split-Bregman method, which determines a global minimizer of the energy functional. 
Acknowledgments Nawal Houhou was supported by Swiss National Science Foundation Grant $\sharp 205320$ - 101621, Xavier Bresson was supported by ONR N00014-03-1-0071 and ONR MURI subcontract from Stanford University. Nawal Houhou and Jean-Philippe Thiran would like to thank Prof. Pierre Vandergheynst for interesting discussions on non-local representation. Xavier Bresson would like to thank Prof. Michael Ng for his invitation at the Third International Conference in Scientific Computing and Partial Differential Equation. Finally the authors would like to thank the referees for their constructive comments and the helpful suggestions.

\section{Appendix}

\section{A.1. Shape derivation tool}

We remind hereafter the shape derivative tool proposed by Delfour and Zolesio in [16]. The shape derivative tool basically derives a region-based functional in an elegant and straightforward way. Let us consider a general region-based functional which depends on an artificial time $\tau$ as follows:

$$
F(\Omega(\tau))=\int_{\Omega} f(x, \Omega(\tau)) d x
$$

that we want to optimize w.r.t $\Omega$. The Gâteaux derivative of the functional $F$ in the directions $V$ is given by:

$$
<F^{\prime}(\Omega), V>=\lim _{\tau \rightarrow 0} \frac{F(\Omega(\tau))-F(\Omega)}{\tau},
$$

where $F^{\prime}(\Omega):=\frac{\partial F}{\partial \tau}(\Omega(\tau))$. As it has been shown in [1], the Gâteaux derivative can be expressed as:

$$
<F^{\prime}, V>=\int_{\Omega} \frac{\partial f}{\partial \tau}(x, \Omega, V) d x-\int_{\partial \Omega} f(s, \Omega)(V(s) \cdot \mathscr{N}(s)) d s,
$$

where $\mathscr{N}$ is the unit inward normal to $\partial \Omega$ the boundary of the evolving region $\Omega, d s$ its length/area element. In the case where we have $f(x)=f\left(x, G_{1}(\tau), G_{2}(\tau)\right)$ and

$$
G_{1}(\tau)=\frac{H_{1}(\tau)}{H_{2}(\tau)}, \quad G_{2}(\tau)=\frac{H_{3}(\tau)}{H_{4}(\tau)}
$$

we obtain:

$$
\begin{aligned}
\frac{\partial f}{\partial \tau} & =\frac{\partial f}{\partial G_{1}} \frac{\partial G_{1}}{\partial \tau}+\frac{\partial h}{\partial G_{2}} \frac{\partial G_{2}}{\partial \tau} \\
& =\frac{\partial f}{\partial G_{1}}\left[\frac{\partial G_{1}}{\partial H_{1}} \frac{\partial H_{1}}{\partial \tau}+\frac{\partial G_{1}}{\partial H_{2}} \frac{\partial H_{2}}{\partial \tau}\right]+\frac{\partial f}{\partial G_{2}}\left[\frac{\partial G_{2}}{\partial H_{3}} \frac{\partial H_{3}}{\partial \tau}+\frac{\partial G_{2}}{\partial H_{4}} \frac{\partial H_{4}}{\partial \tau}\right] .
\end{aligned}
$$




\section{A.2. Gâteaux derivative of functional $K L$}

Consider

$$
\begin{aligned}
& K L\left(q_{\text {in }}(\Omega), q_{\text {out }}(\Omega)\right) \\
= & \int_{\mathbb{R}}\left(q_{\text {in }}(I, \Omega)-q_{\text {out }}(I, \Omega)\right)\left(\log q_{\text {out }}(I, \Omega)-\log q_{\text {in }}(I, \Omega)\right) d I,
\end{aligned}
$$

where

$$
\begin{aligned}
& q_{\text {in }}(I, \Omega(\tau))=G_{1}(\tau)=\frac{\int_{\Omega(\tau)} K(I(x)-I(\hat{x})) d \hat{x}}{\int_{\Omega(\tau)} d \hat{x}}=\frac{H_{1}(\tau)}{H_{2}(\tau)}, \\
& q_{\text {out }}(I, \Omega(\tau))=G_{2}(\tau)=\frac{\int_{\Omega_{0} \backslash \Omega(\tau)} K(I(x)-I(\hat{x})) d \hat{x}}{\int_{\Omega_{0} \backslash \Omega(\tau)} d \hat{x}}=\frac{H_{3}(\tau)}{H_{4}(\tau)}, \\
& h(\tau)=G_{1}(\tau) \log \left(\frac{G_{1}(\tau)}{G_{2}(\tau)}\right)+G_{2}(\tau) \log \left(\frac{G_{2}(\tau)}{G_{1}(\tau)}\right) .
\end{aligned}
$$

Consequently,

$$
\begin{aligned}
\frac{\partial h}{\partial \tau}= & \frac{\partial h}{\partial G_{1}}\left[\frac{1}{H_{2}}\left(-\int_{\partial \Omega(\tau)} K(I(x)-I(s))(V \cdot N) d s\right)\right. \\
& \left.+\left(-\frac{H_{1}}{H_{2}^{2}}\right)\left(-\int_{\partial \Omega(\tau)}(V \cdot N) d s\right)\right] \\
& +\frac{\partial h}{\partial G_{2}}\left[\frac{1}{H_{4}}\left(\int_{\partial \Omega(\tau)} K(I(x)-I(s))(V \cdot N) d s\right)\right. \\
& \left.+\left(-\frac{H_{3}}{H_{4}^{2}}\right)\left(\int_{\partial \Omega(\tau)}(V \cdot N) d s\right)\right] .
\end{aligned}
$$

We can deduce that

$$
\begin{aligned}
\frac{\partial h}{\partial \tau}= & \frac{1}{|\Omega|}\left(1-\frac{q_{\text {out }}(I(x), \Omega)}{q_{\text {in }}(I(x), \Omega)}+\log \frac{q_{\text {in }}(I(x), \Omega)}{q_{\text {out }}(I(x), \Omega)}\right) \\
& \times\left(\int_{\partial \Omega(\tau)}\left(-K(I(x)-I(s))+q_{\text {in }}(I(x), \Omega)\right)(V \cdot N) d s\right) \\
& +\frac{1}{\left|\Omega_{0} \backslash \Omega\right|}\left(1-\frac{q_{\text {in }}(I(x), \Omega)}{q_{\text {out }}(I(x), \Omega)}+\log \frac{q_{\text {out }}(I(x), \Omega)}{q_{\text {in }}(I(x), \Omega)}\right) \\
& \times\left(\int_{\partial \Omega(\tau)}\left(K(I(x)-I(s))-q_{\text {out }}(I(x), \Omega)\right)(V . N) d s\right) .
\end{aligned}
$$

Finally, the Gâteaux derivative of functional (A.4) is:

$$
<K L^{\prime}, V>=\int_{\mathbb{R}} \frac{\partial h}{\partial \tau}(x, \Omega, V) d x .
$$




\section{References}

[1] G. Aubert, M. Barlaud, O. Faugeras, and S. Jehan-Besson. Image Segmentation using Active Contours: Calculus of Variations or Shape Gradients. SIAM Applied Mathematics, 63(6):21282154, 2003.

[2] G. Aubert and P. Kornprobst. Mathematical Problems in Image Processing: Partial Differential Equations and the Calculus of Variations. Springer, 2001.

[3] J.F. Aujol, G. Gilboa, T. Chan, and S. Osher. Structure-Texture Image Decomposition Modeling, Algorithms, and Parameter Selection. International Journal of Computer Vision, 67(1):111-136, 2006.

[4] X. Bresson and T.F. Chan. Non-local Unsupervised Variational Image Segmentation Models, UCLA CAM Report 08-67, 2008.

[5] X. Bresson, S. Esedoglu, P. Vandergheynst, J.P. Thiran, and S. Osher. Fast global minimization of the active contour/snake model. Journal of Mathematical Imaging and Vision, 28(2):151167, June 2007.

[6] X. Bresson, P. Vandergheynst, and J.-P. Thiran. Multiscale Active Contours. International Journal of Computer Vision, 70(3):197-211, 2006.

[7] P. Brodatz. Textures: A photographic album for Artists and Designers. New York, NY, Dover Publication, 1996.

[8] T. Brox, J. Weickert, B. Burgeth, and P. Mrázek. Nonlinear structure tensors. Image Vision Comput., 24(1):41-55, 2006.

[9] A. Buades, B. Coll, and J.M. Morel. A review of image denoising algorithms, with a new one. Multiscale Modeling \& Simulation, 4(2):490-530, 2005.

[10] V. Caselles, R. Kimmel, and G. Sapiro. Geodesic Active Contours. International Journal of Computer Vision, 22(1):61-79, 1997.

[11] A. Chambolle. An Algorithm for Total Variation Minimization and Applications. Journal of Mathematical Imaging and Vision, 20(1-2):89-97, 2004.

[12] T.F. Chan, S. Esedoglu, and M. Nikolova. Algorithms for Finding Global Minimizers of Image Segmentation and Denoising Models. SIAM Journal on Applied Mathematics, 66(5):16321648, 2006.

[13] T.F. Chan, B.Y. Sandberg, and L.A. Vese. Active contours without edges for vector-valued images. J. Visual Communication Image Representation, 11(2):130-141, June 2000.

[14] T.F. Chan and L.A. Vese. Active Contours Without Edges. IEEE Transactions on Image Processing, 10(2):266-277, 2001.

[15] Y. Chen, H.D. Tagare, S. Thiruvenkadam, F. Huang, D. Wilson, K.S. Gopinath, R.W. Briggsand, and E.A. Geiser. Using Prior Shapes in Geometric Active Contours in a Variational Framework. International Journal of Computer Vision, 50(3):315-328, 2002.

[16] M.C. Delfour and J.P. Zolésio. Shapes and Geometries: Analysis, Differential Calculus, and Optimization. Advances in Design and Control, SIAM, 2001.

[17] A. Efros and W.T. Freeman. Image quilting for texture synthesis and transfer. Proceedings of SIGGRAPH 2001, pages 341-346, August 2001.

[18] A. Efros and T. Leung. Texture Synthesis by Non-Parametric Sampling. IEEE International Conference on Computer Vision, 2:10-33, 1999.

[19] L.C. Evans and R.F. Gariepy. Measure Theory and Fine Properties of Functions. Studies in advanced Mathematics, CRC Press, 1992.

[20] D. Freedman and T. Zhang. Active contours for tracking distributions. IEEE Transactions on Image Processing, 13(4):518- 526, April 2004.

[21] G. Gilboa and S. Osher. Nonlocal Linear Image Regularization and Supervised Segmentation. 
SIAM Multiscale Modeling and Simulation (MMS), 6(2):595?30, 2007.

[22] G. Gilboa and S. Osher. Nonlocal Operators with Applications to Image Processing. CAM Report 07-23, 2007.

[23] E. Giusti. Minimal Surfaces and Functions of Bounded Variation. Birkhauser, Basel, 1985.

[24] T. Goldstein, X. Bresson, and S. Osher. Geometric Applications of the Split Bregman Method: Segmentation and Surface Reconstruction. CAM Report 09-06, 2009.

[25] A. Gray. Modern Differential Geometry of Curves and Surfaces with Mathematica. CRC Press, Inc., Boca Raton, FL, USA, 1996.

[26] A. Herbulot, S. Jehan-Besson, M. Barlaud, and G. Aubert. Shape Gradient For Multi-Modal Image Segmentation Using Joint Intensity Distributions. In International Workshop on Image Analysis for Multimedia Interactive Services (WIAMIS), 2004.

[27] N. Houhou, J-P. Thiran, and X. Bresson. Fast Texture Segmentation Model based on the Shape Operator and Active Contour. In Computer Vision and Pattern Recognition, 2008.

[28] A.K. Jain and F. Farrokhnia. Unsupervised Texture Segmentation Using Gabor Filters. In IEEE International Conference on Systems, Man and Cybernetics, pages 14-19, 1990.

[29] S. Jehan-Besson, M. Barlaud, and G. Aubert. DREAM2S: Deformable Regions driven by an Eulerian Accurate Minimization Method for Image and Video Segmentation. International Journal of Computer Vision, 53(1):45-70, 2003.

[30] S. Jehan-Besson, M. Barlaud, G. Aubert, and O. Faugeras. Shape Gradients for Histogram Segmentation using Active Contours. IEEE International Conference on Computer Vision, 1:408415, 2003.

[31] M. Kass, A. Witkin, and D. Terzopoulos. Snakes: Active Contour Models. International Journal of Computer Vision, 1(4):321-331, 1987.

[32] S. Kichenassamy, A. Kumar, P. Olver, A. Tannenbaum, and A.J. Yezzi. Conformal Curvature Flows: From Phase Transitions to Active Vision. In Archive for Rational Mechanics and Analysis, volume 134, pages 275-301, 1996.

[33] R. Kimmel, R. Malladi, and N. Sochen. Images as Embedded Maps and Minimal Surfaces: Movies, Color, Texture, and Volumetric Medical Images. International Journal of Computer Vision, 39(2):111-129, 2000.

[34] E. Kreyszig. Differential Geometry. Paperback, 1991.

[35] T.S. Lee. Image representation using 2d gabor wavelets. IEEE Transactions on Pattern Analysis and Machine Intelligence, 18(10):959-971, 1996.

[36] L. Liang, C. Liu, Y.Q. Xu, B. Guo, and H.Y. Shum. Real-time texture synthesis by patch-based sampling. ACM Trans. Graph., 20(3):127-150, 2001.

[37] S.G. Mallat. A theory for multiresolution signal decomposition: The wavelet representation. IEEE Transactions on Pattern Analysis and Machine Intelligence, 11(7):674-693, July 1989.

[38] S. Marcelja. Mathematical description of the response of simple cortical cells. J. Optical Soc. Am., 70:1297-1300, 1980.

[39] D. Martin, C. Fowlkes, D. Tal, and J. Malik. A database of human segmented natural images and its application to evaluating segmentation algorithms and measuring ecological statistics. In Proceedings of IEEE Computer Society Conference on Computer Vision and Pattern Recognition, volume 2, pages 416-423, July 2001.

[40] S. Osher and J.A. Sethian. Fronts Propagating with Curvature-Dependent Speed: Algorithms Based on Hamilton-Jacobi Formulations. Journal of Computational Physics, 79(1)(12-49), 1988.

[41] N. Paragios and R. Deriche. Geodesic Active Regions: A New Paradigm to Deal with Frame Partition Problems in Computer Vision. Journal of Visual Communication and Image Representation, 13(1-2):249-268, 2002. 
[42] N. Paragios and R. Deriche. Geodesic Active Regions and Level Set Methods for Supervised Texture Segmentation. International Journal of Computer Vision, 46(3):223-247, 2002.

[43] E. Parzen. On the Estimation of a Probability Density Function and the Mode. Annals of Mathematical Statistics, 33(3):1065?076, 1962.

[44] M. Rousson, T. Brox, and R. Deriche. Active unsupervised texture segmentation on a diffusion based feature space. In Proceedings of IEEE Computer Society Conference on Computer Vision and Pattern Recognition, pages 699-704, 2003.

[45] M. Rousson and R. Deriche. A Variational Framework for Active and Adaptative Segmentation of Vector Valued Images. In Workshop on Motion and Video Computing (WMVC), pages 56-61, 2002.

[46] L. I. Rudin, S. Osher, and E. Fatemi. Nonlinear Total Variation Based Noise Removal Algorithms. Physica D, 60(1-4):259 - 268, 1992.

[47] C. Sagiv, N. Sochen, and Y.Y. Zeevi. Integrated active contours for texture segmentation. IEEE Transactions on Image Processing, 15(6):1633-1646, June 2006.

[48] G. Sapiro. Color snakes. Comput. Vis. Image Underst., 68(2):247-253, 1997.

[49] Simon Setzer. Split bregman algorithm, douglas-rachford splitting and frame shrinkage. In SSVM, volume 5567 of Lecture Notes in Computer Science, pages 464-476. Springer, 2009.

[50] N. Sochen, R. Kimmel, and R. Malladi. A General Framework For Low Level Vision. IEEE Transactions on Image Processing, 7(3):310 - 318, 1998.

[51] X.-C. Tai and C. Wu. Augmented lagrangian method, dual methods and split bregman iteration for rof model. In SSVM, volume 5567 of Lecture Notes in Computer Science, pages 502-513. Springer, 2009.

[52] L. Vese and T. Chan. A Multiphase Level Set Framework for Image Segmentation Using the Mumford and Shah Model, UCLA CAM Report 01-25, 2001.

[53] Z. Wang and B.C. Vemuri. An affine invariant tensor dissimilarity measure and its applications to tensor-valued image segmentation. In IEEE International Conference on Computer Vision and Pattern Recognition, pages 228-233, 2004.

[54] J. Weickert. A review of nonlinear diffusion filtering. Proceedings of the International Conference on Scale-Space Theory in Computer Vision, pages 3-28, 1997.

[55] A. Yezzi, A. Tsai, and A. Willsky. A Statistical Approach to Snakes for Bimodal and Trimodal Imagery. In Proceedings of IEEE Computer Society International Conference of Computer Vision, pages 898-903, 1999.

[56] S.C. Zhu and A. Yuille. Region Competition: Unifying Snakes, Region Growing, and Bayes/MDL for Multiband Image Segmentation. IEEE Transactions on Pattern Analysis and Machine Intelligence, 18(9):884-900, 1996. 